\title{
VIVÊNCIAS DE ADOLESCENTES E JOVENS DIAGNOSTICADOS COM DOENÇA RENAL CRÔNICA
}

\section{Cristianne Teixeira Carneiro ${ }^{a^{*}}$, Angelina Monteiro Furtado ${ }^{b^{*}}$, Francis Kanashiro Meneghetti $^{c^{*}}$, Josilene Alves da Rocha Santos ${ }^{\mathrm{d}^{*}}$, Maria Augusta Rocha Bezerra ${ }^{\mathrm{e}^{*}}$, Maria Luzinete Rodrigues da Silva ${ }^{\mathrm{f}^{*}}$, Ruth Cardoso Rocha ${ }^{\mathrm{g}^{*}}$, Silvana Santiago da Rocha ${ }^{\mathrm{h}^{*}}$}

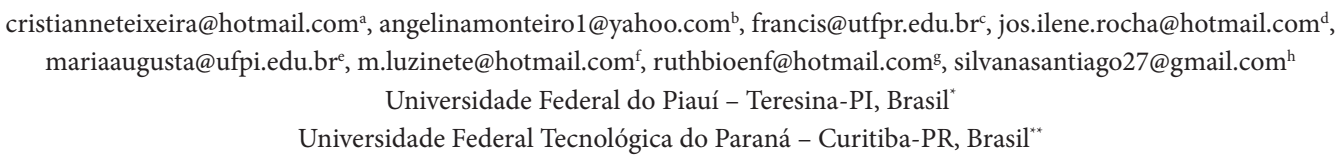

Data do recebimento do artigo: $24 / 01 / 2018$ Data do aceite: $02 / 07 / 2018$

\section{RESUMO}

Introdução: adolescentes e jovens passam por marcantes modificações corporais peculiares da puberdade, acompanhadas por mudanças na esfera psicossocial. Essas mudanças quando associadas a uma doença crônica podem gerar a perda da autoestima e o medo. Objetivo: compreender as vivências de adolescentes e jovens com diagnóstico de Doença Renal Crônica. Método: estudo do tipo exploratório e descritivo, com abordagem predominantemente qualitativa, desenvolvido em uma clínica de nefrologia do município de Floriano (PI), com 13 adolescentes e jovens com faixa etária entre 10 e 29 anos. Os dados foram submetidos à análise de conteúdo. Resultados: a Doença Renal Crônica e o tratamento hemodialítico interferiram na inserção dos participantes no mercado de trabalho e apresentaram repercussões nas suas imagens corporais. A maior parte dos entrevistados revelou incômodo com a presença da fístula arteriovenosa. Entretanto, alguns participantes declararam sentir-se confortáveis com o tratamento e com a Doença Renal Crônica, apontando certa conformidade relacionada à espera do transplante e em relação ao corpo. Conclusão: é preciso que os profissionais de saúde estejam atentos às necessidades dos adolescentes e jovens e dos seus familiares para que possam, através do diálogo, apoiá-los na resolução das dificuldades encontradas.

Palavras-chave: Adolescente; insuficiência renal crônica; diálise renal.

\begin{abstract}
Introduction: adolescents and youngsters undergo remarkable corporal modifications peculiar to puberty, accompanied by changes in the psychosocial sphere. These changes when associated with a chronic illness can lead to loss of self-esteem and fear. Objective: to understand the experiences of adolescents and young people diagnosed with Chronic Renal Disease. Method: an exploratory and descriptive study, with a predominantly qualitative approach, developed in a clinic of nephrology in the municipality of Floriano (PI), with 13 adolescents and young people aged between 10 and 29 years. The data were submitted to content analysis. Results: Chronic Renal Disease and hemodialysis treatment interfered with the insertion of participants in the labor market and had repercussions on their body images. Most of the interviewees were uncomfortable with the presence of the arteriovenous fistula. However, some participants reported feeling comfortable with the treatment and with Chronic Kidney Disease, pointing out some conformity related to waiting for the transplant and in relation to the body. Conclusion: Health professionals must be attentive to the needs of adolescents and their families, so that through dialogue they can support them in solving the difficulties encountered.
\end{abstract}

Keywords: Adolescent; renal insufficiency, chronic; renal dialysis. 


\section{Introdução}

A adolescência é considerada um período de transição entre a infância e a fase adulta, um tempo de rápido amadurecimento físico, cognitivo, social e emocional ${ }^{1}$. Já a juventude é definida como uma fase de transição entre a adolescência e a vida adulta, um momento de preparação. Por conseguinte, adolescentes e jovens passam por marcantes modificações corporais peculiares da puberdade, acompanhadas por mudanças na esfera psicossocial ${ }^{2}$.

Essas mudanças quando associadas a uma doença crônica podem gerar sensações de fracasso, de falta de esperança e de raiva, além de poder desencadear a autocensura, a perda da autoestima e o medo, representando um fardo extra para esses sujeitos ${ }^{2}$. Entre as doenças crônicas que podem acometê-los tem-se a Doença Renal Crônica (DRC), que ocorre devido a alterações na taxa de filtração glomerular e/ou presença de lesão parenquimatosa mantidas por pelo menos três meses ${ }^{3}$.

No mundo, cerca de um milhão e duzentas mil pessoas sobrevivem com DRC sob tratamento hemodialítico. No Brasil, são aproximadamente 70.872 pacientes, dos quais $90,7 \%$ estão em tratamento hemodialítico e 9,3\% em diálise peritoneal, e ainda $1,5 \%$ corresponde a pacientes com idade inferior a 18 anos. A etiologia e a incidência da DRC variam em função da idade. Em crianças antes dos cinco anos, as causas mais frequentes são as malformações (congênitas) do trato urinário, enquanto na faixa etária de cinco a 15 anos prevalecem as doenças renais adquiridas e hereditárias ${ }^{4}$.

No censo publicado em 2008 pela Sociedade Brasileira de Nefrologia (SBN), estimou-se que havia 41.614 pacientes em tratamento dialítico no país, e, destes, 666 estavam com idade menor que 20 anos. Esse censo teve a participação somente de 327 das 684 unidades cadastradas na SBN. Além disso, a taxa de mortalidade em crianças portadoras de DRC em tratamento dialítico é de 30 a $150 \%$ mais alta do que a da população pediátrica geral, e a expectativa de vida para uma criança ou adolescente de zero a 14 anos em diálise é de apenas 20 anos $^{5}$.

A DRC afeta várias dimensões do ser humano, sejam elas físicas, psicológicas, econômicas ou sociais. A situação agrava-se quando se trata de jovens e adolescentes, uma vez que necessitam de cuidados especiais e de apoio familiar efetivo nas tomadas de decisões. Com isso, essa patologia ganha relevância, uma vez que afeta o fluxo de vida cotidiana desses sujeitos, causando uma ruptura com o mundo social em que se encontram inseridos, mudando os seus ritmos de vida e restringindo-os de realizar atividades que antes lhes eram permitidas ${ }^{6}$.

Diariamente, adolescentes e jovens com DRC são submetidos a restrições dietéticas, hídricas, tratamentos difíceis e invasivos, com esquemas medicamentosos complexos e até hospitalizações. Observa-se que eles possuem maior risco de pior desempenho psicossocial que os seus pares saudáveis. Dados da literatura evidenciam que as alterações emocionais nos pacientes com DRC e em seus cuidadores podem persistir também na fase adul$\mathrm{ta}^{7}$. Além disso, trocas negativas familiares, como a superproteção, podem prejudicar a autonomia e autodefesa, o que pode levá-los a apresentar deficiências cognitivas, baixo nível de escolaridade e psicossocial, bem como problemas psiquiátricos, implicando diretamente na sua qualidade de vida ${ }^{8}$.

A partir da compreensão da magnitude do problema e das repercussões que a DRC pode trazer para adolescentes e jovens e suas famílias, objetivou-se compreender as vivências de adolescentes e jovens com diagnóstico de Doença Renal Crônica (DRC).

\section{Método}

Estudo de caráter exploratório e descritivo, com abordagem predominantemente qualitativa. A coleta de dados ocorreu em uma clínica de nefrologia do município de Floriano (PI), em junho do ano de 2015. Participaram do estudo 13 adolescentes e jovens com faixa etária entre 10 e 29 anos. Esta amostra foi determinada mediante a comprovação da saturação, sendo a coleta dos dados interrompida quando as informações fornecidas pelos novos participantes não mais contribuíram expressivamente para a consumação da reflexão teórica desta investigação?

Como critério de inclusão no estudo, estabeleceu-se: adolescentes e jovens que estivessem fazendo tratamento hemodialítico há pelo menos seis meses. 
Como critério de exclusão: adolescentes e jovens que estivessem fazendo tratamento hemodialítico para insuficiência renal em fase aguda da doença.

Neste estudo, adotou-se como referência, para definição de adolescentes, a classificação da Organização Mundial de Saúde (OMS), na qual a adolescência corresponde à faixa etária de 10 a 19 anos, e a classificação do jovem, conforme identificado no Brasil, de acordo com a Política Nacional de Juventude, como o brasileiro que se encontre na faixa etária entre 15 e 29 anos $^{2}$.

Utilizou-se como instrumento para coleta de dados um roteiro semiestruturado, contendo questões abertas e fechadas, abordando os aspectos socioeconômicos e culturais dos entrevistados. Ademais, foi constituído de questões norteadoras relacionadas às vivências de adolescentes e jovens com DRC visando compreender os sentimentos envolvidos na correlação entre o ser adolescente/ jovem, a DRC e a realização da hemodiálise.

Para a análise dos relatos, fundamentou-se na Análise de Conteúdo de Bardin, a qual é um conjunto de técnicas de análise de comunicações, a fim de obter procedimentos sistemáticos e objetivos de descrição do conteúdo das mensagens que permitam a inferência de conhecimentos relativos à percepção destas ${ }^{10}$.

Esta investigação respeitou os aspectos éticos e legais para execução de pesquisas com seres humanos e foi aprovada pelo Comitê de Ética em Pesquisa da Universidade Federal do Piauí por meio do parecer $\mathrm{n}^{0} 1.041 .707 / 2015^{11}$.

\section{RESULTADOS}

Participaram desta pesquisa 13 adolescentes e jovens, em sua maioria do sexo feminino, advindos de municípios dos estados do Maranhão e Piauí. A idade média mais prevalente foi entre 17 e 29 anos. Quanto à escolaridade, o ensino fundamental incompleto foi predominante. A renda familiar variou entre menos de um a três salários mínimos, sendo a mais referida a inferior a um salário mínimo.

Com relação aos aspectos relacionados à DRC e ao tratamento hemodialítico, a maior parte dos adolescentes e jovens foi diagnosticada e iniciou o tratamento na fase da adolescência. Os entre- vistados estão em hemodiálise por um período de um a oito anos e submetem-se à mesma três dias por semana, durante três ou quatro horas por dia. Quanto à ocupação, a maioria dos participantes do estudo não exerce funções empregatícias e apenas quatro estudam. As patologias que mais prevaleceram como fator causal da DRC foram diabetes mellitus, hipertensão arterial sistêmica e glomerulonefrite. Constatou-se ainda uma taxa significativa de participantes que desconhecem a etiologia da sua doença.

No que concerne à categoria Vivências dos Adolescentes e Jovens com DRC, verificou-se que a maioria dos adolescentes e jovens manifesta os sentimentos de tristeza e conformação à espera por um transplante. Segundo o relato a seguir:

"É difícil (cabeça baixa), é muito ruim, mas a gente se conforma. Não tem outro jeito (triste$z a)$, tem que se conformar por enquanto, até esperar um transplante, tá tudo certo [...]" (AJ1)

Evidenciou-se, também, que alguns entrevistados demonstram o sentimento de indiferença em realizar a hemodiálise:

"É (cabeça baixa), eu num acho muito ruim não [...]" (AJ4)

"Normal (cabeça baixa) [...]" (AJ8)

"Não há diferença [...”] (AJ9)

"Olha, pra mimé normal, tranquilo, aqui tem os horários pra vim fazer a hemodiálise, eu venho $[\ldots] "(\mathrm{AJ} 13)$

$\mathrm{Na}$ categoria Alterações da Imagem Corporal Relacionadas à DRC, observou-se que a maioria dos participantes não gosta ou acha desconfortável o seu corpo devido às mudanças relacionadas a essa doença e ao seu tratamento. Os relatos a seguir permitem essa apreensão:

"Acho desconfortável, acho sei lá, difícil [...] foi de ter colocado essa fístula, acho muito ridícula (olhando para o braço) [...]" (AJ1)

"Ah! Mudou muita coisa [...] Mas o que mais foi ter que usar isso aqui (olhando para o braço) [...]" (AJ2)

"O que mudou é que primeiro eu andava com uma blusa e não pode (olhando para o braço) porque tem o aparelho e num pode andar mos- 
trando [...]" (AJ3)

"Mudou! Não gosto das fístulas [...]" (AJ10)

Evidenciou-se outro aspecto, relativo à autoimagem, abordado pelos adolescentes e jovens que revelaram sentir-se confortáveis em relação aos seus corpos pela melhora da sintomatologia da DRC, ao fazer o tratamento hemodialítico. Essas percepções podem ser constatadas no discurso:

Mudei, eu não podia caminhar um pouquim que eu vivia cansada pra morrer, se eu não parasse era arriscado até cair no chão dura, de tanto cansada, doendo as canela, tudo [...] o que mais mudou foi o cansaço que melhorou [...]" (AJ6)

Quanto à categoria Mudanças na Dinâmica de Vida dos Adolescentes e Jovens com a DRC, constatou-se que esta patologia e o tratamento hemodialítico provocam mudanças na dinâmica de vida dos participantes desta pesquisa. A rotina afetada por restrições foi ressaltada nos relatos a seguir:

"Muito, de várias formas [...] Tudo é limitado (pausa, pensando). Não pode fazer isso, não pode fazer aquilo, tem que ter um pouco de paciência [...]" (AJ1)

"Assim, mudou um bocado de coisas, porque o que eu fazia, não posso fazer agora [...]” (AJ6)

"Mudou porque tinha uma vida e agora tá diferente[...]"(AJ12)

Averiguou-se, também, que os adolescentes e jovens vivenciam uma brusca mudança nos seus hábitos de vida e passam a conviver com limitações causadas pelas alterações que interferem em atividades relativas ao estudo e ao trabalho, conforme as falas abaixo:

"Nem trabalho, mudou foi tudo mesmo" [...] (AJ3)

"Mudou! Parei de estudar" [...] (AJ10)

\section{Discussão}

No que diz respeito à categoria Vivências dos Adolescentes e Jovens com DRC, verificou-se que vários fatores repercutem no vivenciar da DRC associada a sua necessidade de tratamento. Dentre eles estão as condições sociais, econômicas, físicas e psíquicas.
O tratamento hemodialítico representa para a maioria das pessoas a continuidade da vida, porém com impacto significativo na qualidade da mesma. A maneira com que elas lidam com as situações são subjetivas e individuais, não existindo um padrão de qualidade de vida, mas formas diferentes de enfrentar a terapêutica ${ }^{12}$.

Os adolescentes e jovens podem manifestar, conforme os relatos, os sentimentos de tristeza, mas também de indiferença, relacionados às dificuldades do tratamento hemodialítico. $\mathrm{O}$ fato de ser um doente renal ou de necessitar de tratamento hemodialítico pode ser considerado, inicialmente, como provocador de sofrimento físico e psíquico, especialmente no momento da descoberta do diagnóstico, entretanto, para cada pessoa, há uma forma de operacionalizar sua avaliação, e a avaliação de um mesmo indivíduo pode variar com o tempo, com o estabelecimento de prioridades ao longo da vida e com as circunstâncias pelas quais a vida pode se modificar ${ }^{13}$.

No que se refere ao sentimento de conformação mencionado nos relatos, a literatura aponta que a despeito da condição e do momento difícil, os adolescentes e jovens conseguem superá-lo. Assim, eles diferem quanto à forma de reagir à doença e a ela se adaptar. Embora enfrentem desespero e medo diante do diagnóstico e tratamento, conseguem sentir-se felizes à medida que se adaptam às mudanças decorrentes da doença ${ }^{14}$.

Dentro da categoria Alterações da Imagem Corporal Relacionadas à DRC, a autoimagem é abordada, sobretudo, pelos adolescentes e jovens, em virtude das alterações em sua aparência física, dada sua condição clínica. Essas alterações se comprovam pela presença do cateter ou da fístula arteriovenosa e dos acessos vasculares utilizados para a hemodiálise ${ }^{15}$.

O sentimento de vergonha associado à aparência física, evidenciado nos discursos, demonstra que há receio dos adolescentes e jovens em imaginar o que as pessoas diriam ou perguntariam a respeito do uso do cateter e das alterações no braço onde se encontra a fístula, o que contribui para uma autoimagem negativa e para o surgimento de sentimentos de inferioridade.

A autoimagem pode ser definida como a visão que cada um tem de si mesmo e surge das interações com o contexto social, com as outras pessoas e consigo mesmo. Os adolescentes e jovens, em razão 
da doença e do tratamento a que são submetidos, apresentam um comprometimento da sua autoimagem, que se reflete em uma sensação de diferença em relação aos seus pares. Encontram-se, ainda, limitados no que se refere à participação em atividades consideradas prazerosas, o que, mais uma vez, pode interferir de forma negativa na autoestima ${ }^{15}$.

Outro aspecto relativo à autoimagem, abordado pelos adolescentes e jovens, foi o sentir-se confortável com o próprio corpo devido à melhora da sintomatologia da DRC, ao fazer o tratamento hemodialítico. Esse evidenciado demonstra que a qualidade de vida desses indivíduos pode apresentar uma relação positiva entre os aspectos da autoimagem e emocionais.

A DRC e o tratamento hemodialítico provocam uma sucessão de situações para os adolescentes e jovens que trazem consequências físicas, psicológicas, com repercussões pessoais, familiares e sociais. Assim, eles vão estabelecendo ao longo do processo de adaptação novas condições, nas quais o indivíduo precisa identificar meios para lidar com o problema renal e com todas as mudanças e limitações que o acompanham, sendo necessário um reaprender a viver de uma maneira mais humana ${ }^{16}$.

Na categoria Mudanças na Dinâmica de Vida dos Adolescentes e Jovens com DRC, as limitações apontadas pelos adolescentes e jovens, mediante as lesões provocadas pela DRC, indicam a necessidade de modificação de suas atividades e rotinas. Esses pacientes passam a ter que disponibilizar uma grande quantidade de seu tempo para realizar o tratamento, três vezes por semana. Para eles, as atividades sociais e outras ocupações referentes ao viver são dispensadas, visto que se prioriza a satisfação de outras necessidades essenciais à sobrevivência ${ }^{17}$.

Nesse sentido, a dificuldade em manter-se produtivo, revelada pelos adolescentes e jovens, demonstra o iminente problema relacionado à vida profissional desses indivíduos. A maioria destes não consegue estabelecer e/ou manter vínculo com o trabalho e a escola, provavelmente devido ao tempo dedicado ao tratamento e à presença de queixas físicas de fraqueza, cansaço, indisposição, mal-estar geral, em especial nos dias de realização da hemodiálise. Este tratamento pode impossibilitar a realização de esforço físico para o trabalho, gerar exaustão e, também, dificuldade para aprender ${ }^{18}$.

Sublinha-se que os adolescentes e jovens apresentaram desestruturação quanto ao funcionamen- to ocupacional nos aspectos relativos à organização do tempo, flexibilidade, mudanças da rotina, objetivos e expectativas para o futuro. Entretanto, as pessoas em hemodiálise não ficam totalmente impossibilitadas de realizar as tarefas do seu cotidiano. Assim, mesmo diante das dificuldades, com o cotidiano alterado por horários de terapia, e, em muitos casos, a perda do trabalho e/ou do estudo, estes sujeitos podem, com a ajuda de uma equipe multiprofissional e de sua rede social: planejar atividades, construir projetos e, assim, fazer algo com significado para si, reconstruindo seu cotidiano ${ }^{18}$.

\section{Conclusão}

O tratamento hemodialítico representa um meio de sobrevivência para os participantes da pesquisa, mas para alguns destes atrapalha sua rotina e dinâmica de vida, especialmente pela necessidade de adesão ao tratamento.

Sobre o nível de instrução, observou-se que a maioria dos adolescentes e jovens possuía até o ensino fundamental incompleto, demonstrando que a DRC implicou na evasão e/ou atraso escolar em virtude da necessidade de realização do tratamento hemodialítico, o que também trouxe repercussões para inserção dos participantes no mercado de trabalho. Este tratamento também apresentou repercussões quanto à imagem corporal do adolescente/jovem, que revelou sentir-se incomodado pela presença da fístula arteriovenosa.

Os diferentes sentimentos identificados nos relatos - tristeza, indiferença, conformação, inferioridade, baixa autoestima - fazem parte dos processos de adaptações vivenciados pelos adolescentes e jovens, e modificam-se durante o processo de conhecimento e enfrentamento da doença.

Dentre a sucessão de mudanças ocorridas na vida dos participantes foram citadas: a incapacidade ou a limitação para a realização das atividades profissionais, físicas e de lazer; e o desconforto em relação ao corpo devido à fistula que implicou na convivência com a família e em manter-se produtivo: estudar/trabalhar. Entretanto, alguns participantes declararam sentir-se confortáveis com o tratamento e a DRC, revelando certa conformação relacionada à espera do transplante e em relação ao corpo.

É preciso que os profissionais de saúde estejam atentos às necessidades dos adolescentes e jovens 
e dos seus familiares para que possam, através do diálogo, apoiá-los na resolução das dificuldades encontradas, percebendo a magnitude de um ser humano que almeja ser atendido ou, pelo menos, ser compreendido em suas expectativas e ser respeitado em sua individualidade. Ações educativas voltadas para a promoção da saúde devem estar dirigidas para socializar o saber já disponível, para favorecer a construção de novas maneiras de viver melhor, apesar das limitações impostas pela doença e pelo tratamento hemodialítico.

\section{Referências}

1. Hockenberry MJ, Wilson D. Wong fundamentos de enfermagem pediátrica. 9. ed. Rio de Janeiro: Elsevier; 2014.

2. Silva EMS, Silva LWS. Impacto da hemodiálise na vida de adolescentes acometidos pela insuficiência renal crônica. Adolesc Saude. 2011;8(1):43-50.

3. Bastos MG, Kirsztajn GM. Doença renal crônica: importância do diagnóstico precoce, encaminhamento imediato e abordagem interdisciplinar estruturada para melhora do desfecho em pacientes ainda não submetidos à diálise. J bras nefrol. 2011;33(1):93-108. doi: http://dx.doi. org/10.1590/S0101-28002011000100013.

4. Pennafort VPS, Queiroz MVO, Jorge MSB. Crianças e adolescentes renais crônicos em espaço educativo-terapêutico: subsídios para o cuidado cultural de enfermagem. Rev Esc Enferm USP. 2012;46(5):1057-65. doi: http://dx.doi.org/10.1590/S0080-62342012000500004.

5. Maciel AC, Miranda JOF. Perfil de crianças e adolescentes com insuficiência renal crônica acompanhados em unidades de nefrologia. J res fundam care online. 2013;5(3):94-103. doi: http://dx.doi.org/10.9789/21755361.2013.v5i3.94-103.

6. Schneider KLK, Martini JG. Cotidiano do adolescente com doença crônica. Texto \& contexto enferm. 2011;20(spe):194-204. doi: http://dx.doi.org/10.1590/ S0104-07072011000500025.

7. Marciano RC, Soares CMB, Diniz JSS, Lima EM, Silva JMP, Canhestro MR, et al. Transtornos mentais e qualidade de vida em crianças e adolescentes com doença renal crônica e em seus cuidadores. J bras nefrol. 2010;32(3):316-22. doi: http://dx.doi.org/10.1590/S010128002010000300014.
8. Sattoe JNT, Jedeloo S, Staa AV. Effective peer-to-peer support for young people with end-stage renal disease: a mixed methods evaluation of Camp COOL. BMC Nephrol. 2013;14:279. doi: https://doi.org/10.1186/14712369-14-279.

9. Fontanella BJB, Luchesi BM, Saidel MGB, Ricas J, Turato ER, Melo DG. Amostragem em pesquisas qualitativas: proposta de procedimentos para constatar saturação teórica. Cad saúde pública. 2011;27(2):388-94. doi: http:// dx.doi.org/10.1590/S0102-311X2011000200020.

10. Bardin L. Análise de conteúdo. 7. ed. São Paulo (SP): Edições 70; 2011.

11. Ministério da Saúde (BR). Resolução no 466, de 12 de dezembro de 2012. Aprova as diretrizes e normas regulamentadoras de pesquisas envolvendo seres humanos. Diário Oficial da União [Internet], Brasília (DF). 13 jul. 2013 [cited 2016 Jul 9]. Seção 1:59. Available from: http://bvsms.saude. gov.br/bvs/saudelegis/cns/2013/res0466_12_12_2012.html

12. Campos CJG, Turato ER. Tratamento hemodialítico sob a ótica do doente renal: estudo clínico qualitativo. Rev bras enferm. 2010;63(5):799-805. doi: http://dx.doi org/10.1590/S0034-71672010000500017.

13. Silva AS, Silveira RS, Fernandes GFM, Lunardi VL, Backes VMS. Percepções e mudanças na qualidade de vida de pacientes submetidos à hemodiálise: Rev bras enferm. 2011;64(5):839-44. doi: http://dx.doi.org/10.1590/S003471672011000500006 .

14. Madeiro AC, Machado PDLC, Bonfim IM, Braqueais AR, Lima FET. Adesão de portadores de insuficiência renal crônica ao tratamento de hemodiálise. Acta paul enferm. 2010;23(4):546-51. doi: http://dx.doi.org/10.1590/S010321002010000400016 .

15. Abreu IS, Kourrouski MFC, Santos DMSS, Bullinger M, Nascimento LC, Lima RAG, et al . Crianças e adolescentes em hemodiálise: atributos associados à qualidade de vida. Rev Esc Enferm USP. 2014;48(4):602-9. doi: http:// dx.doi.org/10.1590/S0080-623420140000400005.

16. Oliveira DG, Guerra WL, Dias SB. Percepção do portador de insuficiência renal crônica acerca da prevenção da doença. Rev Enferm Integ. 2010;3(2):519-32.

17. Ribeiro CDS, Alencar CSM, Feitosa MCD, Mesquita MASB. Percepção do portador de doença renal crônica sobre o tratamento hemodialítico. R Interd. 2013;6(3):36-44.

18. Santos I, Rocha RPF, Berardinelli LMM. Qualidade de vida de clientes em hemodiálise e necessidades de orientação de enfermagem para o autocuidado. Esc Anna Nery Rev Enferm. 2011;15(1):31-8. doi: http://dx.doi. org/10.1590/S1414-81452011000100005.

\section{Como citar este artigo:}

Carneiro CT, Furtado AM, Meneghetti FK, Santos JAR, Bezerra MAR, Silva MLR, Rocha RC, Rocha SS. Vivências de adolescentes e jovens diagnosticados com doença renal crônica. . Rev. Aten. Saúde. 2018;16(57):24-29. 